International Journal of Pure and Applied Mathematics

Volume 114 No. $4 \quad 2017,695-707$

ISSN: 1311-8080 (printed version); ISSN: 1314-3395 (on-line version)

url: http://www.ijpam.eu

doi: 10.12732/ijpam.v114i4.1

\title{
REGULAR $\Gamma$-SO-RINGS
}

\author{
K. Siva Prasad ${ }^{1}$, M. Siva Mala ${ }^{2}$, K. Naga Koteswara Rao ${ }^{3}$ \\ ${ }^{1}$ Department of Mathematics \\ Acharya Nagarjuna University \\ Nagarjuna Nagar-522510, Guntur(D.t), Andhra Pradesh, INDIA \\ ${ }^{2}$ Department of Mathematics \\ V.R. Siddhartha Engineering College \\ Kanuru, Vijayawada-520007, Andhra Pradesh, INDIA \\ ${ }^{3}$ Department of Science \& Humanities \\ Nannapaneni Venkata Rao College of Engineering \& Technology \\ Tenali-522201, Andhra Pradesh, INDIA
}

\begin{abstract}
A $\Gamma$-so-ring is a structure possessing a natural partial ordering, an infinitary partial addition and a ternary multiplication, subject to a set of axioms. The partial functions under disjoint-domain sums and functional composition is a $\Gamma$-so-ring. In this paper we introduce the notions of irreducible, strongly irreducible ideals and a regular $\Gamma$-so-ring and we obtain the characteristics of them.
\end{abstract}

AMS Subject Classification: 16Y60

Key Words: irreducible ideal, strongly irreducible ideal, regular $\Gamma$-so-ring and strongly regular $\Gamma$-so-ring

Received: December 21, 2015

Revised: $\quad$ March 21, 2017

Published: $\quad$ June 7, 2017

(c) 2017 Academic Publications, Ltd. url: www.acadpubl.eu

$\S_{\text {Correspondence author }}$ 


\section{Introduction}

Partially defined infinitary operations occur in the contexts ranging from integration theory to programming language semantics. The general cardinal algebras studied by Tarski in 1949, Housdorff topological commutative groups studied by Bourbaki in 1966, $\Sigma$-structures studied by Higgs in 1980, sum ordered partial monoids \& sum ordered partial semirings studied by Arbib, Manes, Benson[3],[7] and Streenstrup[18] are some of the algebraic structures of the above type.

M. Murali Krishna Rao[11] in 1995 introduced the notion of a $\Gamma$-semiring as a generalization of semirings and $\Gamma$-rings, and extended many fundamental results of semirings and $\Gamma$-rings to $\Gamma$-semirings. In [12] and [13] we introduced the notion of $\Gamma$-so-ring, obtained a necessary and sufficient condition for the quotient $R / \theta$ to be a $\Gamma / \sigma$-so-ring, where $(\theta, \sigma)$ is a congruence relation on $(R, \Gamma)$ and $(\phi, \rho)$-representation of $\Gamma$-so-rings. In [14], [15], [16] and [17], we intoduced the notions of an ideal, a prime ideal and a semiprime ideal in a $\Gamma$ so-ring $R$ and obtained many characteristics of them in $R$. As a continuation, in this paper we introduce the notions of irreducible and strongly irreducible ideals and a regular $\Gamma$-so-ring and we obtain the characteristics of them.

\section{Preliminaries}

In this section we collect important definitions and results from [7], [12], [14],[15], [16], [17] and [18].

Definition 2.1. [7] A partial monoid is a pair $(M, \Sigma)$ where $M$ is a nonempty set and $\Sigma$ is a partial addition defined on some, but not necessarily all families $\left(x_{i}: i \in I\right)$ in $M$ subject to the following axioms:

(i) Unary Sum Axiom. If $\left(x_{i}: i \in I\right)$ is a one element family in $M$ and $I=\{j\}$, then

$\Sigma\left(x_{i}: i \in I\right)$ is defined and equals $x_{j}$.

(ii) Partition-Associativity Axiom. If $\left(x_{i}: i \in I\right)$ is a family in $M$ and $\left(I_{j}: j \in J\right)$ is a

partition of $I$, then $\left(x_{i}: i \in I\right)$ is summable if and only if $\left(x_{i}: i \in I_{j}\right)$ is summable

for every $j$ in $J,\left(\Sigma\left(x_{i}: i \in I_{j}\right): j \in J\right)$ is summable, and

$\Sigma\left(x_{i}: i \in I\right)=\Sigma\left(\Sigma\left(x_{i}: i \in I_{j}\right): j \in J\right)$.

Definition 2.2. $[12]$ Let $(R, \Sigma)$ and $\left(\Gamma, \Sigma^{\prime}\right)$ be two partial monoids. Then 
$R$ is said to be a partial $\Gamma$-semiring if there exists a mapping $R \times \Gamma \times R \longrightarrow R$ (images to be denoted by $x \gamma y$ for $x, y \in R$ and $\gamma \in \Gamma$ ) satisfying the following axioms:

(i) $x \gamma(y \mu z)=(x \gamma y) \mu z$,

(ii) a family $\left(x_{i}: i \in I\right)$ is summable in $R$ implies $\left(x \gamma x_{i}: i \in I\right)$ is summable in $R$

and $x \gamma\left[\Sigma\left(x_{i}: i \in I\right)\right]=\Sigma\left(x \gamma x_{i}: i \in I\right)$,

(iii) a family $\left(x_{i}: i \in I\right)$ is summable in $R$ implies $\left(x_{i} \gamma x: i \in I\right)$ is summable in $R$

and $\left[\Sigma\left(x_{i}: i \in I\right)\right] \gamma x=\Sigma\left(x_{i} \gamma x: i \in I\right)$,

(iv) a family $\left(\gamma_{i}: i \in I\right)$ is summable in $\Gamma$ implies $\left(x \gamma_{i} y: i \in I\right)$ is summable in $R$

and $x\left[\Sigma^{\prime}\left(\gamma_{i}: i \in I\right)\right] y=\Sigma\left(x \gamma_{i} y: i \in I\right)$ for all $x, y, z,\left(x_{i}: i \in I\right)$ in $R$ and $\mu, \gamma$,

$\left(\gamma_{i}: i \in I\right)$ in $\Gamma$.

Definition 2.3. [14] A partial $\Gamma$-semiring $R$ is said to have a left (right) unity if there exists a family $\left(e_{i}: i \in I\right)$ of elements of $R$ and a family $\left(\gamma_{i}: i \in I\right)$ of elements of $\Gamma$ such that $\Sigma_{i} e_{i} \gamma_{i} r=r\left(\Sigma_{i} r \gamma_{i} e_{i}=r\right)$ for any $r$ in $R$.

Definition 2.4. [18] The sum ordering $\leq$ on a partial monoid $(M, \Sigma)$ is the binary relation such that $x \leq y$ if and only if there exists a $h$ in $M$ such that $y=x+h$ for $x, y \in M$.

Definition 2.5. [18] A sum-ordered partial monoid or so-monoid, in short, is a partial monoid in which the sum ordering is a partial ordering.

Definition 2.6. [12] A partial $\Gamma$-semiring $R$ is said be a sum-ordered partial $\Gamma$-semiring (in short $\Gamma$-so-ring) if the partial monoids $R$ and $\Gamma$ are somonoids.

Definition 2.7. [14] Let $R$ be a partial $\Gamma$-semiring, $A$ be a nonempty subset of $R$ and $\Omega$ be a nonempty subset of $\Gamma$. Then the pair $(A, \Omega)$ of $(R, \Gamma)$ is said to be a left (right) partial $\Gamma$-ideal of $R$ if it satisfies the following:

(i) $\left(x_{i}: i \in I\right)$ is a summable family in $R$ and $x_{i} \in A \forall i \in I$ implies $\Sigma_{i} x_{i} \in A$,

(ii) $\left(\alpha_{i}: i \in I\right)$ is a summable family in $\Gamma$ and $\alpha_{i} \in \Omega \forall i \in I$ implies $\Sigma_{i} \alpha_{i} \in \Omega$, and

(iii) for all $x \in R, y \in A$ and $\alpha \in \Omega, x \alpha y \in A(y \alpha x \in A)$.

If $(A, \Omega)$ is both left and right partial $\Gamma$-ideal of a partial $\Gamma$-semiring $R$, then $(A, \Omega)$ is called a partial $\Gamma$-ideal of $R$. If $\Omega=\Gamma$, then $A$ is called a partial ideal of $R$. 
Definition 2.8. [14] Let $R$ be a $\Gamma$-so-ring, $A$ be a nonempty subset of $R$ and $\Omega$ be a nonempty subset of $\Gamma$. Then the pair $(A, \Omega)$ is said to be a left (right) $\Gamma$-ideal of $R$ if it satisfies the following:

(i) $(A, \Omega)$ is a left (right) partial $\Gamma$-ideal of $R$,

(ii) $x \in R$ and $y \in A$ such that $x \leq y$ implies $x \in A$, and

(iii) $\alpha \in \Gamma$ and $\beta \in \Omega$ such that $\alpha \leq \beta$ implies $\alpha \in \Omega$.

If $(A, \Omega)$ is both left and right $\Gamma$-ideal of a $\Gamma$-so-ring $R$, then $(A, \Omega)$ is called a $\Gamma$-ideal of $R$. If $\Omega=\Gamma$, then $A$ is called an ideal of $(R, \Gamma)$.

Definition 2.9. [14] Let $R$ be a $\Gamma$-so-ring. If $A$ and $\Omega$ are subsets of $R$ and $\Gamma$ respectively, then the intersection of all $\Gamma$-ideals of $R$ containing $(A, \Omega)$ is called the $\Gamma$-ideal generated by $(A, \Omega)$ and is denoted by $<(A, \Omega)>$.

If $\Omega=\Gamma$, then $\langle A\rangle$ is the ideal of $(R, \Gamma)$ generated by $A$.

Definition 2.10. [14] A $\Gamma$-so-ring $R$ is said to be a complete $\Gamma$-so-ring if every family of elements in $R$ is summable and every family of elements in $\Gamma$ is summable.

Theorem 2.11. [14] Let $R$ be a complete $\Gamma$-so-ring. If $A$ and $\Omega$ are subsets of $R$ and $\Gamma$ respectively, then the $\Gamma$-ideal generated by $(A, \Omega)$ is the pair $\left(\left\{x \in R \mid x \leq \Sigma_{i} x_{i}+\Sigma_{j} r_{j} \alpha_{j} x_{j}^{\prime}+\Sigma_{k} x_{k}^{\prime \prime} \alpha_{k}^{\prime} r_{k}^{\prime}+\Sigma_{l} r_{l}^{\prime \prime} \alpha_{l}^{\prime \prime} x_{l}^{\prime \prime \prime} \alpha_{l}^{\prime \prime \prime} r_{l}^{\prime \prime \prime}\right.\right.$, where $x_{i}, x_{j}^{\prime}, x_{k}^{\prime \prime}, x_{l}^{\prime \prime \prime} \in A, r_{j}, r_{k}^{\prime}, r_{l}^{\prime \prime}, r_{l}^{\prime \prime \prime} \in R$ and $\left.\alpha_{j}, \alpha_{k}^{\prime}, \alpha_{l}^{\prime \prime}, \alpha_{l}^{\prime \prime \prime} \in \Gamma\right\},\{\beta \in \Gamma \mid \beta \leq$ $\left.\left.\Sigma_{i}^{\prime} \beta_{i}, \beta_{i} \in \Omega\right\}\right)$.

For $n \in \mathbb{N}, \Sigma_{n} a$ means that sum of $n$ copies of $a$.

Remark 2.12. [14] Let $R$ be a complete $\Gamma$-so-ring and $a \in R$. Then the left/right/both sided ideals of $R$ generated by $a$ are

(i) $<a]=\left\{x \in R \mid x \leq \Sigma_{n} a+\Sigma_{j} r_{j} \alpha_{j} a, r_{j} \in R, \alpha_{j} \in \Gamma, n \in \mathbb{N}\right\}$,

(ii) $\left[a>=\left\{x \in R \mid x \leq \Sigma_{n} a+\Sigma_{j} a \alpha_{j} r_{j}, r_{j} \in R, \alpha_{j} \in \Gamma, n \in \mathbb{N}\right\}\right.$,

(iii) $\left\langle a>=\left\{x \in R \mid x \leq \Sigma_{n} a+\Sigma_{j} r_{j} \alpha_{j} a+\Sigma_{k} a \alpha_{k}^{\prime} r_{k}^{\prime}+\Sigma_{l} r_{l}^{\prime \prime} \alpha_{l}^{\prime \prime} a \alpha_{l}^{\prime \prime \prime} r_{l}^{\prime \prime \prime}\right.\right.$, where $r_{j}, r_{k}^{\prime}, r_{l}^{\prime \prime}, r_{l}^{\prime \prime \prime} \in R$ and $\left.\alpha_{j}, \alpha_{k}^{\prime}, \alpha_{l}^{\prime \prime}, \alpha_{l}^{\prime \prime \prime} \in \Gamma, n \in \mathbb{N}\right\}$.

We call $\langle a\rangle$ as the principal ideal generated by $a$.

Remark 2.13. [14] Let $\left\{\left(A_{j}, \Omega_{j}\right) \mid j \in J\right\}$ be a family of $\Gamma$-ideals of a $\Gamma$-so$\operatorname{ring} R$. Then we denote $<\bigcup_{j \in J}\left(A_{j}, \Omega_{j}\right)>$ as $\bigvee_{j \in J}\left(A_{j}, \Omega_{j}\right)$ and thus $\bigvee_{j \in J}\left(A_{j}, \Omega_{j}\right)$ $=\left(\left\{x \in R \mid x \leq \Sigma_{j} x_{j}, x_{j} \in \bigcup_{j \in J} A_{j}\right\},\left\{\beta \in \Gamma \mid \beta \leq \Sigma_{j}^{\prime} \beta_{j}, \beta_{j} \in \bigcup_{j \in J} \Omega_{j}\right\}\right)$. In particular $\left(A_{1} \bigvee A_{2}, \Omega_{1} \bigvee \Omega_{2}\right)=\left(\left\{x \in R \mid x \leq x_{1}+x_{2}, x_{1} \in A_{1}, x_{2} \in A_{2}\right\},\{\beta \in\right.$ $\left.\left.\Gamma \mid \beta \leq \beta_{1}+\beta_{2}, \beta_{1} \in \Omega_{1}, \beta_{2} \in \Omega_{2}\right\}\right)$ is the smallest $\Gamma$-ideal of $R$ containing $\left(A_{i}, \Omega_{i}\right)$ for $i=1,2$. 
Definition 2.14. [14] Let $R$ be a $\Gamma$-so-ring. If $A, B$ are subsets of $R$ and $\Gamma_{1}$ is a subset of $\Gamma$, define $A \Gamma_{1} B$ as the set $\left\{x \in R \mid \exists a_{i} \in A, \gamma_{i} \in \Gamma_{1}, b_{i} \in\right.$ $B, \Sigma_{i} a_{i} \gamma_{i} b_{i}$ exists and $\left.x \leq \Sigma_{i} a_{i} \gamma_{i} b_{i}\right\}$.

If $A=\{a\}$ then we also denote $A \Gamma_{1} B$ by $a \Gamma_{1} B$. If $B=\{b\}$ then we also denote $A \Gamma_{1} B$ by $A \Gamma_{1} b$. Similarly if $A=\{a\}$ and $B=\{b\}$, we denote $A \Gamma_{1} B$ by $a \Gamma_{1} b$ and thus $a \Gamma_{1} b=\left\{x \in R \mid x \leq a \gamma b\right.$ for some $\left.\gamma \in \Gamma_{1}\right\}$.

Remark 2.15. [15] Let $R$ be a $\Gamma$-so-ring with left/right unity and $a, b \in R$. Then $a \in a \Gamma R$ and $b \in R \Gamma b$.

Theorem 2.16. [14] Let $R$ be a complete $\Gamma$-so-ring. If $A$ and $B$ are ideals of $R$ then $A \Gamma B$ is an ideal of $R$. Moreover, $A \Gamma B \subset A \cap B$.

An ideal $A$ of a $\Gamma$-so-ring $R$ is called proper if $A \neq R$.

Definition 2.17. [16] A proper ideal $P$ of a $\Gamma$-so-ring $R$ is said to be prime if and only if for any ideals $A, B$ of $R, A \Gamma B \subseteq P$ implies $A \subseteq P$ or $B \subseteq P$.

Lemma 2.18. [16] Let $R$ be a complete $\Gamma$-so-ring and $P$ be a proper ideal of $R$. Then the following conditions are equivalent:

(i) $P$ is prime

(ii) If $a, b \in R$ such that $\langle a\rangle \Gamma<b\rangle \subseteq P$ then $a \in P$ or $b \in P$.

Lemma 2.19. [16] Let $R$ be a complete $\Gamma$-so-ring and $a \in R$. Then $R \Gamma a \Gamma R=R \Gamma<a>\Gamma R$.

Theorem 2.20. [16] Let $R$ be a complete $\Gamma$-so-ring and $P$ be a proper ideal of $R$. Then the following conditions are equivalent:

(i) $P$ is prime

(ii) If $a, b \in R$ such that $a \Gamma R \Gamma b \subseteq P$ then $a \in P$ or $b \in P$

(iii) If $A_{1}, A_{2}$ are right ideals of $R$ such that $A_{1} \Gamma A_{2} \subseteq P$ then $A_{1} \subseteq P$ or $A_{2} \subseteq P$

(iv) If $B_{1}, B_{2}$ are left ideals of $R$ such that $B_{1} \Gamma B_{2} \subseteq P$ then $B_{1} \subseteq P$ or $B_{2} \subseteq P$.

Lemma 2.21. [16] Let $R$ be a complete $\Gamma$-so-ring and $P$ be a prime ideal of $R$. Then for any $a \in R, R \Gamma a \Gamma R \subseteq P$ implies $a \in P$.

Definition 2.22. [16] Let $R$ be a $\Gamma$-so-ring and $A$ be a nonempty subset of $R$. Then $A$ is said to be an $m$-system of $R$ if for any $a, b \in A$ there exists $r \in R$ and $\alpha, \beta \in \Gamma$ such that $a \alpha r \beta b \in A$.

Theorem 2.23. [16] Let $R$ be a complete $\Gamma$-so-ring and $P$ be a proper ideal of $R$. Then $P$ is prime if and only if $R \backslash P$ is an $m$-system of $R$. 
Definition 2.24. [17] A proper ideal $P$ of a $\Gamma$-so-ring $R$ is said to be semiprime if and only if for any ideal $A$ of $R, A \Gamma A \subseteq P$ implies $A \subseteq P$.

Theorem 2.25. [17] Let $R$ be a complete $\Gamma$-so-ring and $P$ be a proper ideal of $R$. Then the following conditions are equivalent:

(i) $P$ is a semiprime ideal of $R$

(ii) For any $a \in R, a \Gamma R \Gamma a \subseteq P$ implies $a \in P$

(iii) For any right ideal $U$ of $R, U \Gamma U \subseteq P$ implies $U \subseteq P$

(iv) For any left ideal $V$ of $R, V \Gamma V \subseteq P$ implies $V \subseteq P$.

Lemma 2.26. [17] Let $R$ be a complete $\Gamma$-so-ring and $P$ be a semiprime ideal of $R$. Then for any $a \in R, R \Gamma a \Gamma R \subseteq P$ implies $a \in P$.

Definition 2.27. [17] Let $R$ be a $\Gamma$-so-ring and $A$ be a nonempty subset of $R$. Then $A$ is said to be a $p$-system of $R$ if for any $a \in A$ there exists $r \in R$ and $\alpha, \beta \in \Gamma$ such that $a \alpha r \beta a \in A$.

Theorem 2.28. [17] Let $R$ be a complete $\Gamma$-so-ring and $P$ be a proper ideal of $R$. Then $P$ is semiprime if and only if $R \backslash P$ is a $p$-system of $R$.

\section{Irreducible and Strongly Irreducible Ideals}

Definition 3.1. An ideal $P$ of a $\Gamma$-so-ring $R$ is said to be irreducible if and only if for any ideals $H$ and $K$ of $R, P=H \bigcap K$ implies $P=H$ or $P=K$.

Definition 3.2. An ideal $P$ of a $\Gamma$-so-ring $R$ is said to be strongly irreducible if and only if for any ideals $H$ and $K$ of $R, H \cap K \subseteq P$ implies $H \subseteq P$ or $K \subseteq P$.

Clearly every prime ideal of a $\Gamma$-so-ring $R$ is strongly irreducible and every strongly irreducible ideal of $R$ is irreducible. The following is an example of an irreducible ideal which is not a strongly irreducible ideal of a $\Gamma$-so-ring.

Example 3.3. Let $R=\{0, a, b, c, d, e\}$. Define $\Sigma$ on $R$ as

$$
\Sigma_{i} x_{i}=\left\{\begin{array}{l}
x_{j}, \text { if } x_{i}=0 \forall i \neq j, \text { for some } j, \\
d, \text { if }\left(x_{j}=a, x_{k}=b \text { or } x_{j}=b, x_{k}=c \text { for some } j, k\right. \\
\text { and } x_{i}=0 \forall i \neq j, k \\
\text { undefined, otherwise. }
\end{array}\right.
$$

Then $R$ is a so-monoid. 
Let $\Gamma=\left\{0^{\prime}, 1^{\prime}\right\}$. Define $\Sigma^{\prime}$ on $\Gamma$ as

$$
\Sigma_{i}^{\prime} \alpha_{i}=\left\{\begin{array}{l}
1^{\prime}, \text { if } \alpha_{i}=0^{\prime} \forall i \neq j \text { for some } j \\
\text { undefined, otherwise. }
\end{array}\right.
$$

Then $\Gamma$ is a so-monoid.

Define a mapping $R \times \Gamma \times R \rightarrow R$ as follows:

\begin{tabular}{|c|c|c|c|c|c|c|}
\hline $0^{\prime}$ & 0 & $\mathrm{a}$ & $\mathrm{b}$ & $\mathrm{c}$ & $\mathrm{d}$ & $\mathrm{e}$ \\
\hline 0 & 0 & 0 & 0 & 0 & 0 & 0 \\
$\mathrm{a}$ & 0 & 0 & 0 & 0 & 0 & 0 \\
$\mathrm{~b}$ & 0 & 0 & 0 & 0 & 0 & 0 \\
$\mathrm{c}$ & 0 & 0 & 0 & 0 & 0 & 0 \\
$\mathrm{~d}$ & 0 & 0 & 0 & 0 & 0 & 0 \\
$\mathrm{e}$ & 0 & 0 & 0 & 0 & 0 & 0 \\
\hline
\end{tabular}

\begin{tabular}{|c|c|c|c|c|c|c|}
\hline $1^{\prime}$ & 0 & $\mathrm{a}$ & $\mathrm{b}$ & $\mathrm{c}$ & $\mathrm{d}$ & $\mathrm{e}$ \\
\hline 0 & 0 & 0 & 0 & 0 & 0 & 0 \\
$\mathrm{a}$ & 0 & 0 & 0 & 0 & 0 & $\mathrm{a}$ \\
$\mathrm{b}$ & 0 & 0 & 0 & 0 & 0 & $\mathrm{~b}$ \\
$\mathrm{c}$ & 0 & 0 & 0 & 0 & 0 & $\mathrm{c}$ \\
$\mathrm{d}$ & 0 & 0 & 0 & 0 & 0 & $\mathrm{~d}$ \\
$\mathrm{e}$ & 0 & $\mathrm{a}$ & $\mathrm{b}$ & $\mathrm{c}$ & $\mathrm{d}$ & $\mathrm{e}$ \\
\hline
\end{tabular}

Then $R$ is a $\Gamma$-so-ring. For the ideals $A=\{0, a\}, B=\{0, b\}$ and $C=\{0, c\}$ of $R, B \cap C=\{0, b\} \bigcap\{0, c\}=\{0\} \subset A$ and $B \nsubseteq A, C \nsubseteq A$. Hence $A=\{0, a\}$ is not a strongly irreducible ideal of $R$. However the ideal $A=\{0, a\}$ is an irreducible ideal of $R$.

Definition 3.4. Let $R$ be a $\Gamma$-so-ring and $A$ be a non empty subset of $R$. Then $A$ is said to be an i-system of $R$ if and only if for any $a, b \in A$, $<a>\bigcap<b>\bigcap A \neq \emptyset$.

Example 3.5. Let $R=\{0, u, v, x, y, z\}$. Define $\Sigma$ on $R$ as

$$
\Sigma_{i} x_{i}=\left\{\begin{array}{l}
x_{j}, \text { if } x_{i}=0 \forall i \neq j, \text { for some } j, \\
\text { undefined, otherwise. }
\end{array}\right.
$$

Then $R$ is a so-monoid.

Let $\Gamma=\left\{0^{\prime}, 1^{\prime}\right\}$. Define $\Sigma^{\prime}$ on $\Gamma$ as

$$
\Sigma_{i}^{\prime} \alpha_{i}=\left\{\begin{array}{l}
1^{\prime}, \text { if } \alpha_{i}=0^{\prime} \forall i \neq j \text { for some } j \\
\text { undefined, otherwise. }
\end{array}\right.
$$

Then $\Gamma$ is a so-monoid.

Define a mapping $R \times \Gamma \times R \rightarrow R$ as follows:

\begin{tabular}{|c|c|c|c|c|c|c|}
\hline $0^{\prime}$ & 0 & $\mathrm{u}$ & $\mathrm{v}$ & $\mathrm{x}$ & $\mathrm{y}$ & $\mathrm{z}$ \\
\hline 0 & 0 & 0 & 0 & 0 & 0 & 0 \\
$\mathrm{u}$ & 0 & 0 & 0 & 0 & 0 & 0 \\
$\mathrm{v}$ & 0 & 0 & 0 & 0 & 0 & 0 \\
$\mathrm{x}$ & 0 & 0 & 0 & 0 & 0 & 0 \\
$\mathrm{y}$ & 0 & 0 & 0 & 0 & 0 & 0 \\
$\mathrm{z}$ & 0 & 0 & 0 & 0 & 0 & 0 \\
\hline
\end{tabular}

\begin{tabular}{|c|c|c|c|c|c|c|}
\hline $1^{\prime}$ & 0 & $\mathrm{u}$ & $\mathrm{v}$ & $\mathrm{x}$ & $\mathrm{y}$ & $\mathrm{z}$ \\
\hline 0 & 0 & 0 & 0 & 0 & 0 & 0 \\
$\mathrm{u}$ & 0 & $\mathrm{u}$ & 0 & 0 & 0 & $\mathrm{u}$ \\
$\mathrm{v}$ & 0 & 0 & $\mathrm{v}$ & 0 & 0 & $\mathrm{v}$ \\
$\mathrm{x}$ & 0 & 0 & 0 & 0 & 0 & $\mathrm{x}$ \\
$\mathrm{y}$ & 0 & 0 & 0 & 0 & 0 & $\mathrm{y}$ \\
$\mathrm{z}$ & 0 & $\mathrm{u}$ & $\mathrm{v}$ & $\mathrm{x}$ & $\mathrm{y}$ & $\mathrm{z}$ \\
\hline
\end{tabular}


Then $R$ is a $\Gamma$-so-ring. Then the subset $\{0, u\}$ of $R$ is an $i$-system whereas the subset $\{x, y\}$ is not an $i$-system since $\langle x\rangle=\{0, x\},\langle y\rangle=\{0, y\}$ and $<x>\bigcap<y>\bigcap A=\emptyset$.

Theorem 3.6. If $P$ is an ideal of a complete $\Gamma$-so-ring $R$ then the following conditions are equivalent:

(i) $P$ is strongly irreducible

(ii) If $a, b \in R$ such that $\langle a\rangle \bigcap<b>\subseteq P$ then $a \in P$ or $b \in P$

(iii) $R \backslash P$ is an i-system of $R$.

Proof. (i) $\Rightarrow$ (ii): Suppose $P$ is a strongly irreducible ideal of $R$. Let $a, b \in R$ $\ni\langle a\rangle \bigcap<b>\subseteq P$. Since $P$ is strongly irreducible, $\langle a>\subseteq P$ or $\langle b>\subseteq P$. Hence $a \in P$ or $b \in P$.

(ii) $\Rightarrow$ (iii): Suppose $a, b \in R \ni\langle a\rangle \bigcap<b\rangle \subseteq P$ imply $a \in P$ or $b \in P$. Let $a, b \in R \backslash P$. Then $\langle a>\bigcap<b>\not \subset P$. $\Rightarrow<a>\bigcap<b>\bigcap(R \backslash P) \neq \emptyset$. Hence $R \backslash P$ is an $i$-system of $R$.

(iii) $\Rightarrow$ (i): Suppose $R \backslash P$ is an $i$-system of $R$. Let $H, K$ be ideals of $R \ni$ $H \nsubseteq P$ and $K \nsubseteq P$. Then $\exists x \in H$ and $y \in K$ such that $x, y \in R \backslash P$. $\Rightarrow$ $<x>\bigcap<y>\bigcap R \backslash P \neq \emptyset$ (by our assumption). $\Rightarrow \exists z \in<x>\bigcap<y>$ and $z \notin P$. $\Rightarrow z \in H \bigcap K$ and $z \notin P$ and hence $H \bigcap K \nsubseteq P$. Hence $P$ is a strongly irreducible ideal of $R$.

Theorem 3.7. An ideal $P$ of a complete $\Gamma$-so-ring $R$ is prime if and only if it is semiprime and strongly irreducible.

Proof. Suppose $P$ is a prime ideal of $R$. Then $P$ is a semiprime ideal of $R$. Let $H, K$ be ideals of $R \ni H \bigcap K \subseteq P$. Since $H \Gamma K \subseteq H \bigcap K \subseteq P$. Since $P$ is prime, $H \subseteq P$ or $K \subseteq P$ and hence $P$ is a strongly irreducible ideal of $R$.

Conversely, suppose that $P$ is semiprime and strongly irreducible ideal of $R$. Let $H, K$ be ideals of $R \ni H \Gamma K \subseteq P$. Then $(H \bigcap K) \Gamma(H \bigcap K) \subseteq H \Gamma K \subseteq P$. Since $P$ is semiprime, $H \bigcap K \subseteq P$. Since $P$ is strongly irreducible, $H \subseteq P$ or $K \subseteq P$. Hence $P$ is a prime ideal of $R$.

\section{Regular $\Gamma$-so-Rings}

Definition 4.1. Let $R$ be a $\Gamma$-so-ring. An element ' $a$ ' of $R$ is said to be regular if $a \in a \Gamma R \Gamma a$. If every element of $R$ is regular then $R$ is called a regular Г-so-ring. 
Definition 4.2. Let $R$ be a $\Gamma$-so-ring. An element ' $a$ ' of $R$ is said to be strongly regular if there exists $\alpha, \beta \in \Gamma$ and $b \in R$ such that $a \alpha b \beta a=a$. If every element of $R$ is strongly regular then $R$ is called a strongly regular $\Gamma$-so-ring.

Example 4.3. Let $R:=[0,1]$ be the unit interval of real numbers. For any family $\left(x_{i}: i \in I\right)$ in $R$, define $\Sigma_{i} x_{i}=\sup \left\{x_{i} \mid i \in I\right\}$. Then $R$ is a so-monoid. Let $\Gamma:=\mathbb{N} \bigcup\{0\}$, the set of all nonnegative integers. Then $\Gamma$ is a so-monoid with finite support addition. Consider the mapping $(x, \alpha, y) \mapsto \inf \{x, \alpha, y\}$ of $R \times \Gamma \times R$ into $R$. Now $R$ is a $\Gamma$-so-ring. For any $a \in R \exists 1,1 \in \Gamma$ and $a \in R$ such that $a 1 a 1 a=\inf \{a, 1, a, 1, a\}=a$. Hence $R$ is strongly regular $\Gamma$-so-ring.

Lemma 4.4. Let $R$ be a complete $\Gamma$-so-ring with left unity. Then $R$ is regular if and only if $B \Gamma A=A \bigcap B$ for any left ideal $A$ and right ideal $B$ of $R$.

Proof. Suppose $R$ is regular. Let $A$ be a left ideal and $B$ be a right ideal of R. Then $B \Gamma A \subseteq R \Gamma A \subseteq A$ and $B \Gamma A \subseteq B \Gamma R \subseteq B$ and hence $B \Gamma A \subseteq A \cap B$. Let $a \in A \bigcap B$. Since $R$ is regular, $a \in a \Gamma R \Gamma a$. $\Rightarrow a \leq \Sigma_{i} a \alpha_{i} b_{i} \beta_{i} a$ for some $\alpha_{i}, \beta_{i} \in \Gamma, b_{i} \in R$. Since $a \in A$ and $A$ is a left ideal of $R, b_{i} \beta_{i} a \in A . \Rightarrow a \leq$ $\Sigma_{i} a \alpha_{i}\left(b_{i} \beta_{i} a\right), a \in B, \alpha_{i} \in \Gamma, b_{i} \beta_{i} a \in A . \Rightarrow a \in B \Gamma A$. Therefore $A \bigcap B \subseteq B \Gamma A$. Hence $B \Gamma A=A \bigcap B$.

Conversely, suppose that $B \Gamma A=A \bigcap B$ for any left ideal $A$ and right ideal $B$ of $R$. Let $a \in R$. Take $A:=<a$, the principal left ideal generated by $a$ and $B:=[a>$, the principal right ideal generated by $a$. Then $A=A \cap R=$ $R \Gamma A \subseteq R \Gamma a$ and $B=R \bigcap B=B \Gamma R \subseteq a \Gamma R$. Now $a \in A \bigcap B \subseteq R \Gamma a \bigcap a \Gamma R=$ $a \Gamma R \Gamma R \Gamma a \subseteq a \Gamma R \Gamma a$. Therefore $a$ is a regular element in $R$. Hence $R$ is a regular $\Gamma$-so-ring.

Theorem 4.5. Let $R$ be a regular complete $\Gamma$-so-ring with left unity and $P$ be an ideal of $R$. Then $P$ is prime if and only if it is irreducible.

Proof. Suppose $P$ is prime, then by theorem 3.7, $P$ is strongly irreducible and hence $P$ is irreducible. Conversely suppose that $P$ is irreducible. Let $A, B$ be ideals of $R$ such that $A \Gamma B \subseteq P$. Let $x \in(A \bigvee P) \cap(B \bigvee P)$. Since $R$ is regular, by lemma 4.4, $(A \bigvee P) \Gamma(B \bigvee P)=(A \bigvee P) \bigcap(B \bigvee P)$ and hence $x \in(A \bigvee P) \Gamma(B \bigvee P) . \Rightarrow x \leq \Sigma_{i} y_{i} \alpha_{i} z_{i}$ where $y_{i} \in A \bigvee P, \alpha_{i} \in \Gamma, z_{i} \in B \bigvee P$, $i \in I . \Rightarrow x \leq \Sigma_{i}\left[\Sigma_{j}\left(p_{i_{\mathrm{j}}}+a_{i_{\mathrm{j}}}\right)\right] \alpha_{i}\left[\Sigma_{k}\left(p_{i_{\mathrm{k}}}^{\prime}+b_{i_{\mathrm{k}}}\right)\right]$ for some $p_{i_{\mathrm{j}}}, p_{i_{\mathrm{k}}}^{\prime} \in P, a_{i_{\mathrm{j}}} \in$ $A, b_{i_{\mathrm{k}}} \in B . \Rightarrow x \leq \Sigma_{i} \Sigma_{j} \Sigma_{k}\left[p_{i_{\mathrm{j}}} \alpha_{i} p_{i_{\mathrm{k}}}^{\prime}+p_{i_{\mathrm{j}}} \alpha_{i} b_{i_{\mathrm{k}}}+a_{i_{\mathrm{j}}} \alpha_{i} p_{i_{\mathrm{k}}}^{\prime}+a_{i_{\mathrm{j}}} \alpha_{i} b_{i_{\mathrm{k}}}\right]$ and hence $x \in P \bigvee(A \Gamma B)=P . \Rightarrow(A \bigvee P) \bigcap(B \bigvee P)=P$. Since $P$ is irreducible, $A \bigvee P=P$ or $B \bigvee P=P$. $\Rightarrow A \subseteq P$ or $B \subseteq P$. Hence $P$ is a prime ideal of $R$. 
Lemma 4.6. Let $R, R^{\prime}$ be complete $\Gamma$-so-rings and $f: R \longrightarrow R^{\prime}$ be a $\Gamma$-epimorphism. If $R$ is regular then $R^{\prime}$ is also regular.

Proof. Let $a^{\prime} \in R^{\prime}$. Since $f$ is onto, $\exists a \in R \ni f(a)=a^{\prime}$. Since $R$ is regular, $a \in a \Gamma R \Gamma a$. This implies $\exists \alpha_{i}, \beta_{i} \in \Gamma, b_{i} \in R \ni a \leq \Sigma_{i} a \alpha_{i} b_{i} \beta_{i} a . \Rightarrow$ $f(a) \leq f\left(\Sigma_{i} a \alpha_{i} b_{i} \beta_{i} a\right)=\Sigma_{i} f(a) \alpha_{i} f\left(b_{i}\right) \beta_{i} f(a) . \Rightarrow a^{\prime} \in a^{\prime} \Gamma R^{\prime} \Gamma a^{\prime}$. Therefore $a^{\prime}$ is a regular element in $R^{\prime}$. Hence $R^{\prime}$ is a regular $\Gamma$-so-ring.

Theorem 4.7. Let $R$ be a regular complete $\Gamma$-so-ring and $\theta$ be a $\Gamma$ congruence relation on $R$. Then the quotient $\Gamma$-so-ring $R / \theta$ is regular.

Proof. Define a mapping $f: R \rightarrow R / \theta$ by $f(a)=\theta a \forall a \in R$. Now we prove that $f$ is a $\Gamma$-epimorphism. Let $\left(a_{i}: i \in I\right)$ be a (summable) family in $R$. Then $f\left(\Sigma_{i} a_{i}\right)=\theta\left(\Sigma_{i} a_{i}\right)=\overline{\Sigma_{i}}\left(\theta a_{i}\right)=\overline{\Sigma_{i}} f\left(a_{i}\right)$. Now let $a, b \in R$ and $\alpha \in \Gamma$. Then $f(a \alpha b)=\theta(a \alpha b)=(\theta a) \alpha(\theta b)=f(a) \alpha f(b)$. Hence $f$ is a $\Gamma$ homomorphism of $R$ into $R / \theta$. Now for every $\theta a \in R / \theta$, there exists $a \in R$ such that $f(a)=\theta a$. Therefore $f$ is a $\Gamma$-epimorphism. By the Lemma 4.6, $R / \theta$ is a regular $\Gamma$-so-ring.

\section{Strongly Regular $\Gamma$-so-Rings}

Lemma 5.1. Let $R$ be a $\Gamma$-so-ring. If $a$ is a strongly regular element in $R$ then there exists $\alpha, \beta \in \Gamma$ and $b \in R$ such that $a \alpha b \beta a=a$ and $b \beta a \alpha b=b$.

Proof. Since $a$ is a strongly regular element of $R$, by definition $\exists \alpha, \beta \in$ $\Gamma, e \in R \ni a=a \alpha e \beta a$. Take $b:=e \beta a \alpha e$. Then $b \in R$ and $a \alpha b \beta a=$ $a \alpha(e \beta a \alpha e) \beta a=(a \alpha e \beta a) \alpha e \beta a=a \alpha e \beta a=a$. Now $b \beta a \alpha b=(e \beta a \alpha e) \beta a \alpha(e \beta a \alpha e)=$ $e \beta a \alpha e=b$. Hence the lemma.

Definition 5.2. Let $R$ be a $\Gamma$-so-ring and $\alpha \in \Gamma$. An element $a$ of $R$ is said to be an $\alpha$-idempotent if $a \alpha a=a$.

Example 5.3. Consider the $\Gamma$-so-ring $R:=[0,1]$ as in the Example 5.3. Let $0 \neq \alpha \in \Gamma$ and $a \in R$. Then $a \alpha a=\inf \{a, \alpha, a\}=a$. Hence every element of $R$ is an $\alpha$-idempotent of $R$, where $\alpha \neq 0$.

Proposition 5.4. Let $R$ be a strongly regular $\Gamma$-so-ring. Then for any $\alpha \in \Gamma$, there exists an $\alpha$-idempotent element $e$ in $R$. 
Proof. Let $a \in R$ and $\alpha \in \Gamma$. Then $a \alpha a \in R$. Since $R$ is strongly regular, $\exists \beta, \gamma \in \Gamma$ and $b \in R \ni(a \alpha a) \beta b \gamma(a \alpha a)=a \alpha a$ and $b \gamma(a \alpha a) \beta b=b$ (by the Lemma 5.1). Take $e:=a \beta b \gamma a$, then $e \in R$ and $e \alpha e=(a \beta b \gamma a) \alpha(a \beta b \gamma a)=$ $a \beta(b \gamma a \alpha a \beta b) \gamma a=a \beta b \gamma a=e$. Hence $e$ is an $\alpha$-idempotent element in $R$.

Proposition 5.5. Let $I$ be an ideal of a strongly regular $\Gamma$-so-ring $R$. Then $I$ is strongly regular and any ideal $J$ of $I$ is an ideal of $R$.

Proof. Let $I$ be an ideal of a strongly regular $\Gamma$-so-ring $R$. Since $I$ is an ideal of $R, I$ is a sub $\Gamma$-so-ring of $R$. Let $a \in I \subseteq R$. Since $R$ is strongly regular, $\exists \alpha_{1}, \alpha_{2} \in \Gamma$ and $b \in R \ni a=a \alpha_{1} b \alpha_{2} a$ and $b:=b \alpha_{2} a \alpha_{1} b$. Since $a \in I, I$ is an ideal of $R$ so $b \in I$. Therefore $a$ is a strongly regular element in $I$. Hence $I$ is strongly regular. Let $J$ be an ideal of $I$ and let $a \in J, \alpha \in \Gamma, r \in R$. Since $I$ is an ideal of $R, a \alpha r \in I$. Since $I$ is strongly regular, $\exists \alpha_{3}, \alpha_{4} \in \Gamma$ and $d \in R \ni(a \alpha r) \alpha_{3} d \alpha_{4}(a \alpha r)=a \alpha r$. Since $r \alpha_{3} d \alpha_{4} a \alpha r \in I$ and $J$ is an ideal of $I$, $a \alpha\left(r \alpha_{3} d \alpha_{4} a \alpha r\right) \in J . \Rightarrow a \alpha r \in J$. Similarly we can prove that $r \alpha a \in J$. Hence $J$ is an ideal of $R$.

Lemma 5.6. Let $R$ be a complete $\Gamma$-so-ring. If $a=a \alpha b \beta a, \alpha, \beta \in \Gamma$, $b \in R$ then the following are hold in $R$ :

(i) $[a>=a \Gamma R=a \alpha R$,

(ii) $<a]=R \Gamma a=R \beta a$, and

(iii) $\langle a>=R \beta a \alpha R$.

Proof. (i) Note that $\left[a>=\left\{x \in R \mid x \leq \Sigma_{i} a+\Sigma_{i} a \alpha_{i} x_{i}, \alpha_{i} \in \Gamma, x_{i} \in R, i \in\right.\right.$ $I\}$. Since $a=a \alpha b \beta a, a \in a \Gamma R$. Therefore $[a>\subseteq a \Gamma R$. Now let $x \in a \Gamma R$. Then $x \leq \Sigma_{i} a \alpha_{i} x_{i}, \alpha_{i} \in \Gamma, x_{i} \in R, i \in I . \Rightarrow x \in[a>$. Therefore $a \Gamma R \subseteq[a>$. Hence $\left[a>=a \Gamma R\right.$. Now clearly $a \alpha R \subseteq a \Gamma R$. Let $x \in a \Gamma R$. Then $x \leq \Sigma_{i} a \alpha_{i} x_{i}$, $\alpha_{i} \in \Gamma, x_{i} \in R, i \in I . \Rightarrow x \leq \Sigma_{i}(a \alpha b \beta a) \alpha_{i} x_{i} . \Rightarrow x \leq a \alpha\left(b \beta \Sigma_{i} a \alpha_{i} x_{i}\right) . \Rightarrow$ $x \in a \alpha R$. Therefore $a \Gamma R \subseteq a \alpha R$. Hence $[a>=a \Gamma R=a \alpha R$.

Similarly, we prove that $<a]=R \Gamma a=R \beta a$ and $<a>=R \beta a \alpha R$.

Proposition 5.7. Let $R$ be a complete $\Gamma$-so-ring. If an element a of $R$ is strongly regular then there exists a $\beta$-idempotent $e \in R$ such that $[a>=e \beta R$.

Proof. Suppose $a$ is a strongly regular element in $R$. Then $\exists \alpha, \beta \in \Gamma$ and $b \in R \ni a=a \alpha b \beta a$. Take $e=a \alpha b$. Then $e \in R$ and $e \beta e=(a \alpha b) \beta(a \alpha b)=$ $(a \alpha b \beta a) \alpha b=a \alpha b=e$. Also $e \beta a=a \alpha b \beta a=a$. Then by the Lemma 5.6, $[a>=a \alpha R=e \beta a \alpha R=e \beta(a \alpha R) \subseteq e \beta R$. Let $x \in e \beta R$. Then $x \leq e \beta d$ for some $d \in R$. $\Rightarrow x \leq e \beta d=(a \alpha b) \beta d=a \alpha(b \beta d) \in a \alpha R=[a>$. $\Rightarrow x \in[a>$. Hence $[a>=e \beta R$. 
Proposition 5.8. Let $R$ be a strongly regular $\Gamma$-so-ring, $R^{\prime}$ be any $\Gamma$-soring. Let $f$ be a $\Gamma$-epimorphism of $R$ onto $R^{\prime}$ and $\alpha$ in $\Gamma$. If $e^{\prime}$ is an $\alpha$-idempotent in $R^{\prime}$, then $f^{-1}\left(e^{\prime}\right)$ contains an $\alpha$-idempotent of $R$.

Proof. Suppose $e^{\prime}$ is an $\alpha$-idempotent in $R^{\prime}$. Then $e^{\prime} \alpha e^{\prime}=e^{\prime}$. Since $f$ is onto, $\exists e \in R \ni f(e)=e^{\prime}$. Since $R$ is strongly regular eae $\in R, \exists \beta, \gamma \in \Gamma$ and $c \in R \ni(e \alpha e) \beta c \gamma(e \alpha e)=e \alpha e$ and $c \gamma(e \alpha e) \beta c=c$. Now $(e \beta c \gamma e) \alpha(e \beta c \gamma e)=$ $e \beta(c \gamma e \alpha e \beta c) \gamma e=e \beta c \gamma e$ and hence $e \beta c \gamma e$ is an $\alpha$-idempotent in $R . \Rightarrow f(e \beta c \gamma e)=$ $f(e) \beta f(c) \gamma f(e)=e^{\prime} \beta f(c) \gamma e^{\prime}=e^{\prime} \alpha e^{\prime} \beta f(c) \gamma e^{\prime} \alpha e^{\prime}=f(e \alpha e) \beta f(c) \gamma f(e \alpha e)=$ $f($ e $\alpha e \beta c \gamma e \alpha e)=f($ e $\alpha e)=e^{\prime} \alpha e^{\prime}=e^{\prime}$. Thus $f^{-1}\left(e^{\prime}\right)$ contains an $\alpha$-idempotent of $R$.

Definition 5.9. The centre of a $\Gamma$-so-ring $R$ is defined to be the set $\{a \in R \mid a \gamma x=x \gamma a \forall x \in R, \gamma \in \Gamma\}$, and is denoted by $C(R)$. Thus $C(R)=\{a \in R \mid a \gamma x=x \gamma a \forall x \in R, \gamma \in \Gamma\}$.

Theorem 5.10. The centre $C(R)$ of a $\Gamma$-so-ring $R$ is a sub $\Gamma$-so-ring of $R$.

Proof. Let $\left(a_{i}: i \in I\right)$ be a summable family in $R$ such that $a_{i} \in C(R)$ $\forall i \in I$. Then $\Sigma_{i} a_{i}$ exists in $R$ and $a_{i} \gamma x=x \gamma a_{i} \forall x \in R$ and $\forall \gamma \in \Gamma$. Now for any $\gamma \in \Gamma$ and $x \in R,\left(\Sigma_{i} a_{i}\right) \gamma x=\Sigma_{i}\left(a_{i} \gamma x\right)=\Sigma_{i}\left(x \gamma a_{i}\right)=x \gamma\left(\Sigma_{i} a_{i}\right)$ and hence $\Sigma_{i} a_{i} \in C(R)$. Now let $a, b \in C(R)$ and $\alpha \in R$. Then $a \gamma x=x \gamma a \forall x \in R$ and $\gamma \in \Gamma$. For any $\gamma \in \Gamma$ and $x \in R,(a \alpha b) \gamma x=a \alpha(b \gamma x)=(b \gamma x) \alpha a=(x \gamma b) \alpha a=$ $x \gamma(b \alpha a)=x \gamma(a \alpha b)$ and hence $a \alpha b \in C(R)$. Hence $C(R)$ is a sub $\Gamma$-so-ring of $R$.

Definition 5.11. An element $x$ in a $\Gamma$-so-ring $R$ is said to be nilpotent if there exists a positive integer $n$ such that $(x \alpha)^{n} x=0$ for all $\alpha$ in $\Gamma$ where $(x \alpha)^{n} x=x \alpha x \alpha \ldots x \alpha x$

Theorem 5.12. Let $R$ be a strongly regular $\Gamma$-so-ring. If all idempotents of $R$ are in its centre then $R$ has no nonzero nilpotent elements.

Proof. Suppose all idempotent elements of $R$ are in $C(R)$. Let $a \in R$. Since $R$ is strongly regular, $\exists \alpha, \beta \in \Gamma$ and $x \in R \ni a \alpha x \beta a=a$. Now $(a \alpha x) \beta(a \alpha x)=$ $(a \alpha x \beta a) \alpha x=a \alpha x$ and hence $a \alpha x$ is a $\beta$-idempotent. $\Rightarrow a \alpha x \in C(R)$. Therefore $a=a \alpha x \beta a=(a \alpha x) \beta a=a \beta(a \alpha x)=(a \beta a) \alpha x$. Suppose $a$ is nilpotent. Then $(a \alpha)^{n} a=0$ for all $\alpha \in \Gamma$, for some positive integer $n$. $\Rightarrow a=(a \beta a) \alpha x=$ $a \beta(a \beta a \alpha x) \alpha x=\ldots . .=(a \beta)^{n} a \alpha(x \alpha x) \alpha \ldots(x \alpha x)=0$. Thus $R$ has no nonzero nilpotent elements. 


\section{References}

[1] G.V.S. Acharyulu, Matrix representable So-rings, Semigroup Forum, Springer-Verlag, 46(1993), 31-47, doi: 10.1007/BF02573542.

[2] G.V.S. Acharyulu, A Study of Sum-Ordered Partial Semirings, Doctoral thesis, Andhra University, 1992.

[3] M.A. Arbib, E.G. Manes, Partially Additive Categories and Flow-diagram Semantics, Journal of Algebra, 62(1980), 203-227.

[4] T.K. Dutta, S.K. Sardar, Semiprime ideals and irreducible ideals of $\Gamma$-semirings, Novi Sad J. Math., 30(1)(2000), 97-108.

[5] T.K. Dutta, S.K. Sardar, On the Operator Semirings of a $\Gamma$-semiring, Southeast Asian Bulletin of Mathematics, 26(2)(2002), 215-225.

[6] Jonathan S. Golan. : Semirings and their Applications, Kluwer Academic Publishers, 1999.

[7] E.G. Manes, D.B. Benson, The Inverse Semigroup of a Sum-Ordered Partial Semiring, Semigroup Forum, 31(1985), 129-152, doi: 10.1007/BF02572645.

[8] M. Murali Krishna Rao, $\Gamma$-semirings-I, Southeast Asian Bulletin of Mathematics, 19(1)(1995), 49-54.

[9] M. Murali Krishna Rao, $\Gamma$-semirings-II, Southeast Asian Bulletin of Mathematics, 21(1997), 281-287.

[10] M. Murali Krishna Rao, The Jacobson Radical of a $\Gamma$-semiring, Southeast Asian Bulletin of Mathematics, 23(1999), 127-134.

[11] M. Murali Krishna Rao, Г-Semirings, Doctoral thesis, Andhra University, 1995.

[12] M. Siva Mala, K. Siva Prasad, Partial $\Gamma$-Semirings, Southeast Asian Bulletin of Mathematics, 38(6)(2014), 873-885.

[13] M. Siva Mala, K. Siva Prasad, $(\phi, \rho)$-Representation of $\Gamma$-So-Rings, Iranian Journal of Mathematical Sciences and Informatics, 10(1)(2015), 103-119, doi: 10.7508/ijmsi.2015.01.008.

[14] M. Siva Mala, K. Siva Prasad, Ideals of Sum-Ordered partial $\Gamma$-Semirings, Southeast Asian Bulletin of Mathematics, 40(2016), 413-426.

[15] K. Siva Prasad, M. Siva Mala, P.V. Srinivasa Rao, Green's Relations in Partial $\Gamma-$ Semirings, International Journal of Algebra and Statistics(IJAS), 2(2)(2013), 21-28, doi: 10.20454/ijas.2013.755.

[16] M. Siva Mala, K. Siva Prasad, Prime Ideals of $\Gamma$-So-rings, International Journal of Algebra and Statistics(IJAS), 3(1)(2014), 1-8, doi: 10.20454/ijas.2014.774.

[17] M. Siva Mala, K. Siva Prasad, Semiprime Ideals of $\Gamma$-So-rings, International Journal of Algebra and Statistics(IJAS), 3(1)(2014), 26-33, doi: 10.20454/ijas.2014.814.

[18] M.E. Streenstrup, Sum-Ordered Partial Semirings, Doctoral thesis, Graduate school of the University of Massachusetts, Feb 1985 (Department of Computer and Information Science).

[19] P.V. Srininvasa Rao, Ideals of Sum Ordered Partial semirings, International Journal of Computational Cognition(IJCC), 7(2)(2009), 59-64.

[20] P.V. Srinivasa Rao, Ideal Theory of Sum-Ordered Partial Semirings, Doctoral thesis, Acharya Nagarjuna University, 2011. 
